Research Article

\title{
Rehabilitation Nursing Intervention Can Improve Dysphagia and Quality of Life of Patients Undergoing Radiotherapy for Esophageal Cancer
}

\author{
Xiange Zeng, ${ }^{1}$ Ling $\mathrm{Li}^{2}{ }^{2}$ Wenjing Wang, ${ }^{1}$ and Lihui Zhu $\mathbb{D}^{1}$ \\ ${ }^{1}$ Department of Gastroenterology, The Second Affiliated Hospital, Hengyang Medical School, University of South China, \\ Hengyang, China \\ ${ }^{2}$ Digestive Endoscopy Center, The Second Affiliated Hospital, Hengyang Medical School, University of South China, \\ Hengyang, China \\ Correspondence should be addressed to Lihui Zhu; anzagou5@163.com
}

Received 3 June 2021; Accepted 29 July 2021; Published 5 August 2021

Academic Editor: Alamgeer Yuchi

Copyright (C) 2021 Xiange Zeng et al. This is an open access article distributed under the Creative Commons Attribution License, which permits unrestricted use, distribution, and reproduction in any medium, provided the original work is properly cited.

\begin{abstract}
Objective. To seek the improvement of rehabilitation nursing intervention on dysphagia and quality of life of patients with esophageal cancer undergoing radiotherapy. Methods. A total of 109 patients with esophageal cancer undergoing radiotherapy were selected as research objects. According to the random number table, they were separated into the control group (CG) and intervention group (IG), with 45 cases in CG and 64 cases in IG. In CG, patients were given routine nursing intervention, while those in IG were given rehabilitation nursing intervention. After intervention, the degree of acute radiation injury and the improvement of swallowing function were observed to compare the self-nursing ability, quality of life, and incidence of complications between the two groups. Results. The degree of injury in CG was heavier than that in IG. The improvement of swallowing function in IG was better than that in CG. The scores of self-nursing ability and life quality in IG were higher than those in CG, with statistically significant differences $(p<0.05)$. The incidence of complications in IG was obviously lower than that in CG $(p<0.05)$. Conclusion. Rehabilitation nursing intervention can ameliorate dysphagia, improve the quality of life, and reduce the incidence of complications for patients with esophageal cancer undergoing radiotherapy. It is worthy of clinical application.
\end{abstract}

\section{Introduction}

Esophageal cancer, also known as esophagus cancer, is a malignant tumor disease that develops in esophageal epithelial tissue, mostly occurring in people over 40 years old [1]. Its early symptoms are not obvious, but patients will show progressive dysphagia and other symptoms in the middle and late stages, which seriously threatens their life health [2]. The occurrence and distribution of esophageal cancer are related to many factors, including age, gender, race, living environment, and geographical distribution [3-5]. China is a country with a high incidence of esophageal cancer. Due to the improvement of diet and living habits, the incidence of esophageal cancer in China has declined in recent years, but its mortality rate remains high $[6,7]$. Because there are no obvious clinical symptoms in the early stage of esophageal cancer, $80 \%$ of sufferers have entered the middle and late stages when they are diagnosed, often missing the best opportunity to radically cure it [8].

Surgery [9], radiopharmaceutical therapy (radiotherapy) [10], and chemotherapy [11] are the basic methods to treat esophageal cancer. If timely and effective treatment measures are given at the early stage of the patient's disease development, it is highly curative, so it is very necessary to give effective surgical treatment in the early and middle stage of patients' disease development [12]. However, most patients lose the chance of operation because of the location of tumor or the rapid progress of disease, so radiotherapy needs to be given to patients with advanced disease. However, the spread of cancer cells cannot be controlled when the radiotherapy measures are implemented, so the incidence of adverse reactions is higher after treatment $[13,14]$, which 
leads to greater psychological pressure of patients, directly affects their psychological state and quality of life, and has a certain negative impact on the therapeutic effect $[15,16]$. Moreover, radiation injury to esophageal mucosa is one of the most common side effects of radiotherapy, which can lead to mucosal edema, congestion, ulcer, and erosion causing patients to feel esophageal burning, esophageal swelling and pain, and then suffering from dysphagia, swallowing pain, pain behind the sternum, and other symptoms when eating [17-19]. Therefore, the implementation of scientific and effective rehabilitation training and health guidance is conducive to improve the swallowing function for esophageal cancer patients with difficulty in opening mouth after radiotherapy, so as to supplement enough nutrition and water to improve the body's resistance, which is of great significance for the rehabilitation of disease and psychology. In this study, rehabilitation nursing was mainly used to intervene patients with esophageal cancer undergoing radiotherapy and observe its application effect.

\section{Materials and Methods}

2.1. Research Objects. A total of 109 patients with esophageal cancer were selected as the research objects, and the patients were admitted to hospital from June 2019 to June 2020. They were randomly divided into CG and IG in accordance with the random number table. There were 45 cases in CG, including 29 men and 16 women, with an mean age of $47.68 \pm 3.51$ years old. There were 64 cases in IG, including 37 men and 27 women, with a mean age of $48.05 \pm 3.57$ years old.

Inclusion criteria are as follows: all of them met the diagnostic criteria of esophageal cancer, and all patients received radiotherapy.

Exclusion criteria are as follows: comorbid with other tumors, cognitive impairment, and severe hepatic insufficiency.

There was no statistically significant difference in baseline data in both groups $(p>0.05)$, which was comparable. In both groups, patients and their families have affixed informed consent, and this research has been ratified by the ethics committee of our hospital.

2.2. Methods. In both groups, patients received radiotherapy. During radiotherapy, patients in CG were provided with corresponding nursing measures according to the routine nursing process of esophageal cancer. The vital signs, blood routine, and systemic reactions of patients were closely observed, and symptomatic treatment was given in time. The ward was ventilated regularly to keep the air fresh, and the ward was kept quiet. The ward was regularly disinfected, and patients with low leucocyte or infection should be placed in protective isolation. The patient was placed in a comfortable position and kept the respiratory tract smooth.

In IG, patients were treated with rehabilitation nursing intervention. Specific measures: (1) preparatory work. Medical staff explained the importance and positive role of rehabilitation exercise to patients and their families and introduced the content of rehabilitation exercise, so that patients and their families could get a basic understanding, so as to establish a good attitude towards treatment and preparation. (2) Rehabilitation exercises: rehabilitators and nursing staff assisted patients in rehabilitation exercises, including mouth opening exercises, neck massage, oral organ coordination training, and direct feeding training. (3) Oral and pharyngeal nursing. The swallowing therapeutic apparatus with frequency of $30-80 \mathrm{~Hz}$, wave width of $700 \mathrm{~ms}$, and current intensity of $0-25 \mathrm{~mA}$ was used for treatment, and it could be adjusted according to the specific condition and tolerance of patients. The patient was treated for $30 \mathrm{~min} /$ time, once/d, and continued for 2 weeks. (4) Psychological nursing. When patients received radiotherapy, they needed to be enlightened by nursing staff to relieve their anxiety and fear and give appropriate psychological comfort to enhance their self-confidence to overcome the disease.

2.3. Outcome Measures. Mucosal injury degree: it was judged according to the grading standard of acute radiation injury of tumor cooperative organization in radiotherapy, which was divided into four grades, and the severity was decreased in turn according to the grades. Grade 4: the patient had a large area of ulcer, fistula, and perforation, and the pain was intense. Grade 3: the patient suffered from severe dysphagia with complications such as weight loss and severe dehydration. The patient needed to be fed by intravenous fluids infusion or nasal feeding. Grade 2: the patient suffered from moderate dysphagia with labor pains. The patient might take a liquid diet or rely on some anesthetic to relieve the pain. Grade 1: the patients suffered from mild dysphagia and could take semiliquid food, and the epidermal anesthetics could be used to relieve discomfort. Grade 0: there was no symptom.

Swallowing X-ray video (VFSS) was used to evaluate the improvement of swallowing function. Cure: the swallowing time was normal, without error aspiration, residual barium meal, and epiglottic vallecula. Effective: the swallowing time was shortened by more than $1 / 2$ compared with before treatment, and there was no error aspiration, residual barium meal, and epiglottic vallecula. Ineffective: the swallowing time was shortened by less than $1 / 2$ compared with before treatment, and there was error aspiration, residual barium meal, and epiglottic vallecula, or even the patient was unable to swallow.

Self-nursing ability: the self-nursing ability scale ESCA was applied to assess the self-nursing ability of the patients in both groups. The scale included four parts: self-concept, selfcare responsibility, self-care skills, and health knowledge cognition, with a total of 43 items. Each item was scored by 1-4 grades. The higher the score, the higher the self-nursing ability.

The quality of life core scale (EORTC QLQ-C30) was applied to evaluate patients' life quality, which was divided into somatic function, role function, cognitive impairment, emotional function, and society function, with 30 items in total. The higher the score, the better the life quality of patients. 
Incidence of complications: the complications mainly included esophagitis, tracheitis, esophageal fistula, and hemorrhage.

2.4. Statistical Methods. SPSS22.0 statistical software was applied to analyze the research data. The quantitative data were represented by mean number \pm standard deviation $(x \pm s)$, and the $t$-test was applied. The enumeration data were represented by rate (\%), and the $\chi^{2}$ test was applied. The difference was statistically significant with $p<0.05$.

\section{Results}

3.1. Degree of Mucosal Injury in the Two Groups. The number of patients with grade 0 and grade 1 mucosal injury in IG was higher than that in CG, and the number of grade 2, grade 3 , and grade 4 was lower than that in CG. The difference was statistically significant $(p<0.05)$, indicating that the degree of injury in CG was heavier than that in IG (Table 1).

\subsection{Improvement of Swallowing Function in the Two Groups.} Observing the improvement of swallowing function in both groups, the findings showed that the total response rate in IG $(82.8 \%)$ was higher than $57.8 \%$ in CG, and the difference was statistically significant $(p<0.05)$ (Table 2$)$.

3.3. Self-Care Ability of Patients in Both Groups. The selfnursing ability of patients was observed in the two groups. Before intervention, there was no obvious difference in scores of self-concept, self-care responsibility, self-care skills, and health knowledge cognition in both groups $(p<0.05)$. After intervention, the scores of patients in both groups increased, and the scores of patients in IG were obviously higher than those in CG $(p<0.05)$ (Table 3$)$.

3.4. Life Quality of Patients in the Two Groups. There was no significant difference in EORTC QLQ-C30 scores in both groups before intervention. After intervention, the EORTC QLQ-C30 scores of the two groups were higher than those before intervention, and the scores in IG were obviously higher than those in CG $(p<0.05)$ (Figure 1).

3.5. Incidence of Complications in Both Groups. In IG, there were 1 case with esophagitis, 2 cases with tracheitis, 1 case with esophageal fistula, and 0 cases with bleeding, with a total of 4 cases $(6.3 \%)$. In CG, there were 3 cases with esophagitis, 3 cases with tracheitis, 2 cases with esophageal fistula, and 2 cases with bleeding, with a total of 10 cases (22.2\%). There was a statistical difference in the incidence of complications in both groups $(p<0.05)$ (Table 4$)$.

\section{Discussion}

With the change of people's diet structure, the prevalence of esophageal cancer is increasing year by year. Radiotherapy has become the main treatment for esophageal cancer due to less trauma than surgery and less restrictions by surrounding tissues and trachea [20-22]. However, this treatment can lead to serious side effects and increase the psychological burden of patients $[23,24]$. Therefore, it is of positive significance to carry out necessary nursing intervention for patients with esophageal cancer during chemotherapy to improve the clinical prognosis.

In this study, rehabilitation nursing was used to intervene patients undergoing radiotherapy for esophageal cancer. The results showed that the total effective rate of improving swallowing function in IG was $82.8 \%(53 / 64)$, significantly higher than $57.8 \%$ in the control group, which was consistent with the findings in stroke patients with hemiplegia [25]. Radiotherapy can cause different degrees of radiotherapy reaction during the treatment of esophageal cancer. The main toxic side effect is acute radiation esophagitis. The clinical manifestation of acute radiation injury is pain aggravated by eating or burning sensation behind sternum, and its incidence increases with the increase of dose, which has adverse effects on the treatment effect, life quality, and survival rate of patients. The results showed that rehabilitation nursing intervention could effectively reduce the degree of acute radiation injury. The scores of self-nursing ability and life quality in IG were higher than those in CG, and the incidence of complications in IG was $6.3 \%(4 / 64)$, obviously lower than $22.2 \%(10 / 45)$ in CG. Rehabilitation nursing is designed to make every link in the nursing process continuous and systematic and provide high-efficiency and high-quality nursing services for patients, so as to effectively relieve the psychological and physiological pressure of patients and improve the quality of nursing work. Through rehabilitation exercise, oropharyngeal care, psychological intervention, and other measures, the correct and effective rehabilitation nursing in the radiotherapy process can improve the clinical symptoms and reduce the psychological burden of patients, so that patients take the initiative to cooperate with radiotherapy to improve compliance. The results suggested that rehabilitation nursing intervention could obviously ameliorate patients' selfnursing ability and their life quality. Patients with esophageal cancer should be evaluated and take functional exercise as early as possible. Rehabilitation nursing intervention measures (the main content is to understand their condition and psychological characteristics, formulate nursing plans, give health education, rehabilitation training, specialized nursing, and psychological counseling) can significantly ameliorate the degree of swallowing difficulties of patients and prevent the development and progression of complications. This study also has some limitations. For example, the effect of patient disease types on the results of the study 
TABLe 1: Degree of mucosal injury in the two groups.

\begin{tabular}{|c|c|c|c|c|c|}
\hline & Grade 0 & Grade 1 & Grade 2 & Grade 3 & Grade 4 \\
\hline CG $(n=45)$ & $1(2.2)$ & $10(22.2)$ & $22(48.9)$ & $8(17.8)$ & $4(8.9)$ \\
\hline IG $(n=64)$ & $9(14.1)^{*}$ & $37(57.8)^{*}$ & $15(23.4)^{*}$ & $3(4.7)$ & $0(0)^{*}$ \\
\hline$\chi^{2} / t$ & 4.4451 & 13.6510 & 7.6331 & 1.6881 & 5.9061 \\
\hline$P$ & 0.0350 & 0.0002 & 0.0057 & 0.1939 & 0.0151 \\
\hline
\end{tabular}

${ }^{*}$ Comparison with the CG group $(p<0.05)$.

TABLE 2: Improvement of swallowing function in the two groups.

\begin{tabular}{lcccc}
\hline & Cure & Effective & Ineffective & Total effective rate \\
\hline CG $(n=45)$ & $5(11.1)$ & $21(45.7)$ & $19(42.2)$ & $26(57.8)$ \\
IG $(n=64)$ & $16(25.0)$ & $37(57.8)$ & $10(17.2)$ & $53(82.8)^{*}$ \\
$\chi^{2} / t$ & & & 8.3011 \\
$P$ & & & 0.0040 \\
\hline
\end{tabular}

${ }^{*}$ Comparison with the CG group $(p<0.05)$.

TABLe 3: Self-care ability of patients in the two groups.

\begin{tabular}{|c|c|c|c|c|c|c|c|c|}
\hline & \multicolumn{2}{|c|}{ Before intervention } & \multirow{2}{*}{$\chi^{2} / t$} & \multirow{2}{*}{$P$} & \multicolumn{2}{|c|}{ After intervention } & \multirow{2}{*}{$\chi^{2} / t$} & \multirow{2}{*}{$P$} \\
\hline & CG $(n=45)$ & IG $(n=64)$ & & & CG $(n=45)$ & IG $(n=64)$ & & \\
\hline Self-concept & $24.75 \pm 4.26$ & $24.94 \pm 4.03$ & 0.2367 & 0.8133 & $29.03 \pm 5.12$ & $32.68 \pm 5.39^{*}$ & 3.5530 & 0.0005 \\
\hline Self-care responsibility & $25.37 \pm 3.97$ & $25.56 \pm 4.11$ & 0.2410 & 0.8100 & $29.77 \pm 5.69$ & $34.82 \pm 5.61^{*}$ & 4.6000 & $<0.0001$ \\
\hline Self-care skills & $25.77 \pm 4.51$ & $25.93 \pm 4.22$ & 0.1894 & 0.8501 & $29.06 \pm 5.97$ & $36.81 \pm 6.23^{*}$ & 6.5046 & $<0.0001$ \\
\hline Health knowledge cognition & $26.08 \pm 4.87$ & $26.21 \pm 4.93$ & 0.1362 & 0.8919 & $30.19 \pm 6.06$ & $36.49 \pm 6.17^{*}$ & 5.2871 & $<0.0001$ \\
\hline
\end{tabular}

${ }^{*}$ Comparison with the CG group after intervention $(p<0.05)$.

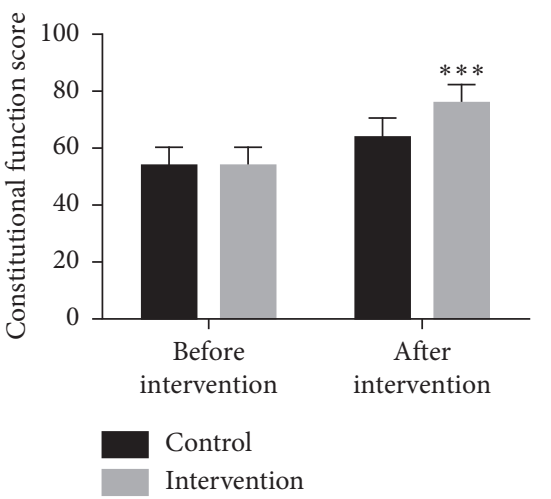

(a)

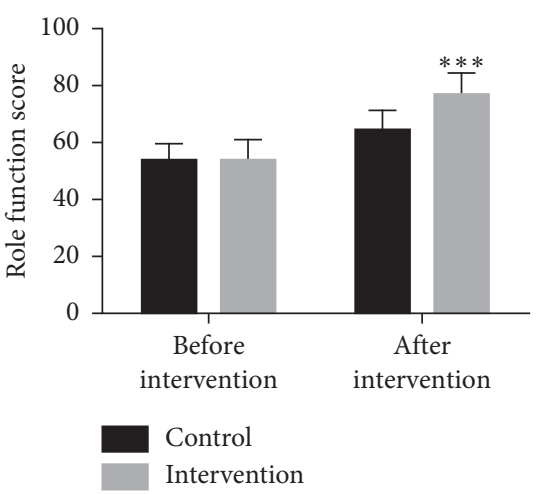

(b)

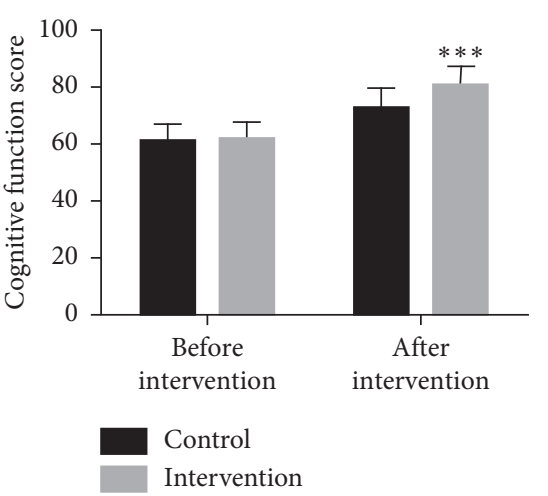

(c)

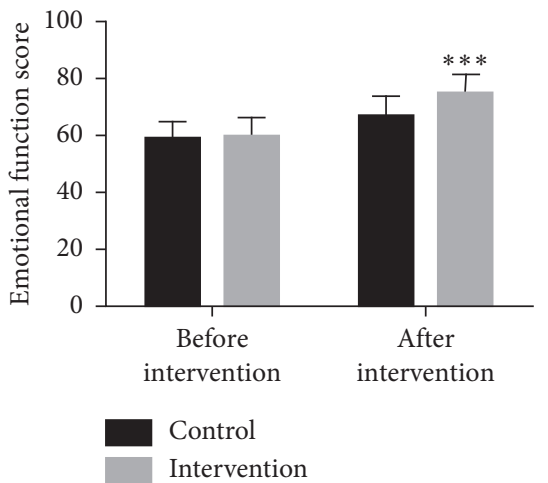

(d)

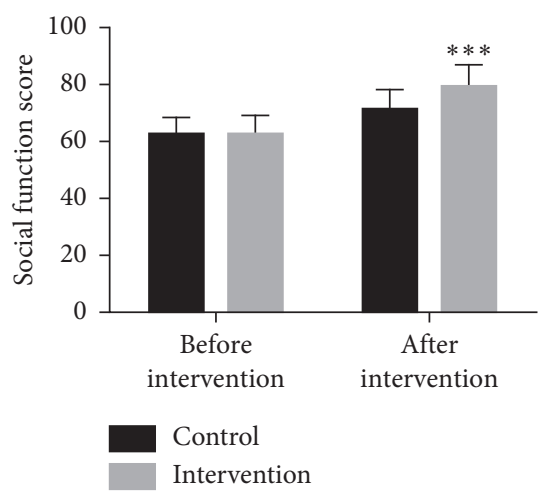

(e)

FIgure 1: Quality of life of patients in the two groups. (a) Physical function scores of patients in the two groups. (b) Role function scores of patients in the two groups. (c) Cognitive function scores of patients in the two groups. (d) Emotional function scores of patients in the two groups. (e) Social function scores of patients in the two groups. Compared with the CG, $\left.{ }^{* * *} p<0.0001\right)$. 
TABLE 4: Incidence of complications in the two groups.

\begin{tabular}{lccccc}
\hline & Esophagitis & Tracheitis & Esophageal fistula & Hemorrhage & Overall incidence \\
\hline CG $(n=45)$ & $3(6.7)$ & $3(6.7)$ & $2(4.4)$ & $2(4.4)$ & $0(0)$ \\
IG $(n=64)$ & $1(1.6)$ & $2(3.1)$ & $1(1.6)$ & $4(6.3)^{*}$ \\
$\chi^{2} / t$ & & & 6.0211 \\
$P$ & & & & 0.0141 \\
\hline
\end{tabular}

${ }^{*}$ Comparison with the CG group $(p<0.05)$.

was not considered, and the small number of included cases may lead to bias in the results.

\section{Conclusion}

In conclusion, rehabilitation nursing intervention has a remarkable effect on dysphagia in patients undergoing radiotherapy for esophageal cancer, which can ameliorate their pain symptoms, improve the quality of life, and reduce the incidence of complications. It is worthy of popularization and application in radiotherapy.

\section{Data Availability}

The data used to support the findings of this study are included within the article.

\section{Conflicts of Interest}

The authors declare that there are no conflicts of interest.

\section{Acknowledgments}

This study was financially supported by Hunan Health Commission Major Project (A2017014).

\section{References}

[1] W. Short Matthew, G. Burgers Kristina, and T. Fry Vincent, "Esophageal cancer," American Family Physician, vol. 95, pp. 22-28, 2017.

[2] S. Joseph, M. Zfass Alvin, S. Mitchell et al., "Early esophageal cancer: a gastroenterologist's disease," Digestive Diseases and Sciences, vol. 64, pp. 3048-3058, 2019.

[3] H. Fang-Liang and S.-J. Yu, "Esophageal cancer: risk factors, genetic association, and treatment," Asian Journal of Surgery, vol. 41, pp. 210-215, 2018.

[4] G. K. Malhotra, U. Yanala, A. Follet, M. Vijayakumar, and C. Are, "Global trends in esophageal cancer," Journal of Surgical Oncology, vol. 115, no. 5, pp. 564-579, 2017.

[5] D. R. S. Middleton, L. Bouaoun, R. Bray et al., "Esophageal cancer male to female incidence ratios in Africa: a systematic review and meta-analysis of geographic, time and age trends," Cancer Epidemiology, vol. 53, pp. 119-128, 2018.

[6] R. Chen, R. S. Zheng, S. W. Zhang et al., "Analysis of incidence and mortality of esophageal cancer in China, 2015," Zhonghua Yu Fang Yi Xue Za Zhi, vol. 53, pp. 1094-1097, 2019.

[7] Y. Y. Li, L. B. Du, X. Q. Hu et al., "A suggested framework for conducting esophageal cancer screening in China," Journal of Digestive Diseases, vol. 19, pp. 722-729, 2018.

[8] Y. Horie, T. Yoshio, K. Yoshimizu et al., "Diagnostic outcomes of esophageal cancer by artificial intelligence using convolutional neural networks," Gastrointestinal Endoscopy, vol. 89, pp. 25-32, 2019.

[9] H. Kikuchi and H. Takeuchi, "Future perspectives of surgery for esophageal cancer," Annals of Thoracic and Cardiovascular Surgery, vol. 24, no. 5, pp. 219-222, 2018.

[10] J. Lyu, T. Li, C. Xie et al., "Enteral nutrition in esophageal cancer patients treated with radiotherapy: a Chinese expert consensus 2018," Future Oncology, vol. 15, no. 5, pp. 517-531, 2019.

[11] G. Y. Ku, "Systemic therapy for esophageal cancer: chemotherapy," Chinese Clinical Oncology, vol. 6, no. 5, p. 49, 2017.

[12] S. Makoto and K. Hiroyuki, "Current status and future prospects for esophageal cancer treatment," Annals of Thoracic and Cardiovascular Surgery, vol. 23, pp. 1-11, 2017.

[13] C. Zhu, S. Wang, Y. You, K. Nie, and Y. Ji, "Risk factors for esophageal fistula in esophageal cancer patients treated with radiotherapy: a systematic review and meta-analysis," Oncology Research and Treatment, vol. 43, no. 1-2, pp. 34-41, 2020.

[14] H. Song, F. Fang, U. Lu et al., "Waiting time for cancer treatment and mental health among patients with newly diagnosed esophageal or gastric cancer: a nationwide cohort study," BMC Cancer, vol. 17, no. 1, p. 2, 2017.

[15] V. Lancellotta, F. Cellini, B. De Sanctis et al., "The role of palliative interventional radiotherapy (brachytherapy) in esophageal cancer: an AIRO (Italian Association of Radiotherapy and Clinical Oncology) systematic review focused on dysphagia-free survival," Brachytherapy, vol. 19, pp. 104-110, 2020.

[16] K. Terufumi, N. Keiji, S. Keisuke et al., "Palliative radiotherapy and chemoradiotherapy in stage IVA/B esophageal cancer patients with dysphagia," International Journal of Clinical Oncology, vol. 23, pp. 1076-1083, 2018.

[17] W. Li, B. Zheng, S. Zhang, H. Chen, W. Zheng, and C. Chen, "Feasibility and outcomes of modified enhanced recovery after surgery for nursing management of aged patients undergoing esophagectomy," Journal of Thoracic Disease, vol. 9, no. 12, pp. 5212-5219, 2017.

[18] Y. Lian, X. Niu, H. Cai et al., "Clinicopathological significance of c-MYC in esophageal squamous cell carcinoma," Tumour Biology: The Journal of the International Society for Oncodevelopmental Biology and Medicine, vol. 39, Article ID $1010428317715804,2017$.

[19] J. Y. Cho, D. Y. Cheung, T. J. Kim, and J. K. Kim, “A case of esophageal squamous cell carcinoma in situ arising from esophageal squamous papilloma," Clinical Endoscopy, vol. 52, no. 1 , pp. 72-75, 2019.

[20] M. . Stefan, C. Mickael, N. Nadja et al., "Early esophageal cancer: the significance of surgery, endoscopy, and chemoradiation," Annals of the New York Academy of Sciences, vol. 1434, pp. 115-123, 2018.

[21] C. C. Abnet, M. Arnold, and W.-Q. Wei, "Epidemiology of esophageal squamous cell carcinoma," Gastroenterology, vol. 154, no. 2, pp. 360-373, 2018. 
[22] X. C. Li, M. Y. Wang, M. Yang et al., "A mutational signature associated with alcohol consumption and prognostically significantly mutated driver genes in esophageal squamous cell carcinoma," Annals of Oncology, vol. 29, no. 4, pp. 938-944, 2018.

[23] L. Zheng, S. Liu, H. Chen et al., "[Relationship between lifestyle and dietary habits and the occurrence of squamous cell carcinoma in different subsites of the esophagus]," Wei Sheng Yan Jiu, vol. 48, pp. 757-764, 2019.

[24] N.-B. Chen, B. Qiu, J. Zhang et al., "Intensity-modulated radiotherapy versus three-dimensional conformal radiotherapy in definitive chemoradiotherapy for cervical esophageal squamous cell carcinoma: comparison of survival outcomes and toxicities," Cancer Research and Treatment, vol. 52, no. 1, pp. 31-40, 2020.

[25] P. Jelvehgaran, D. M. de Bruin, G. Borst et al., "Optical coherence tomography to detect acute esophageal radiationinduced damage in mice: a validation study," Journal of Biophotonics, vol. 12, Article ID e201800440, 2019. 